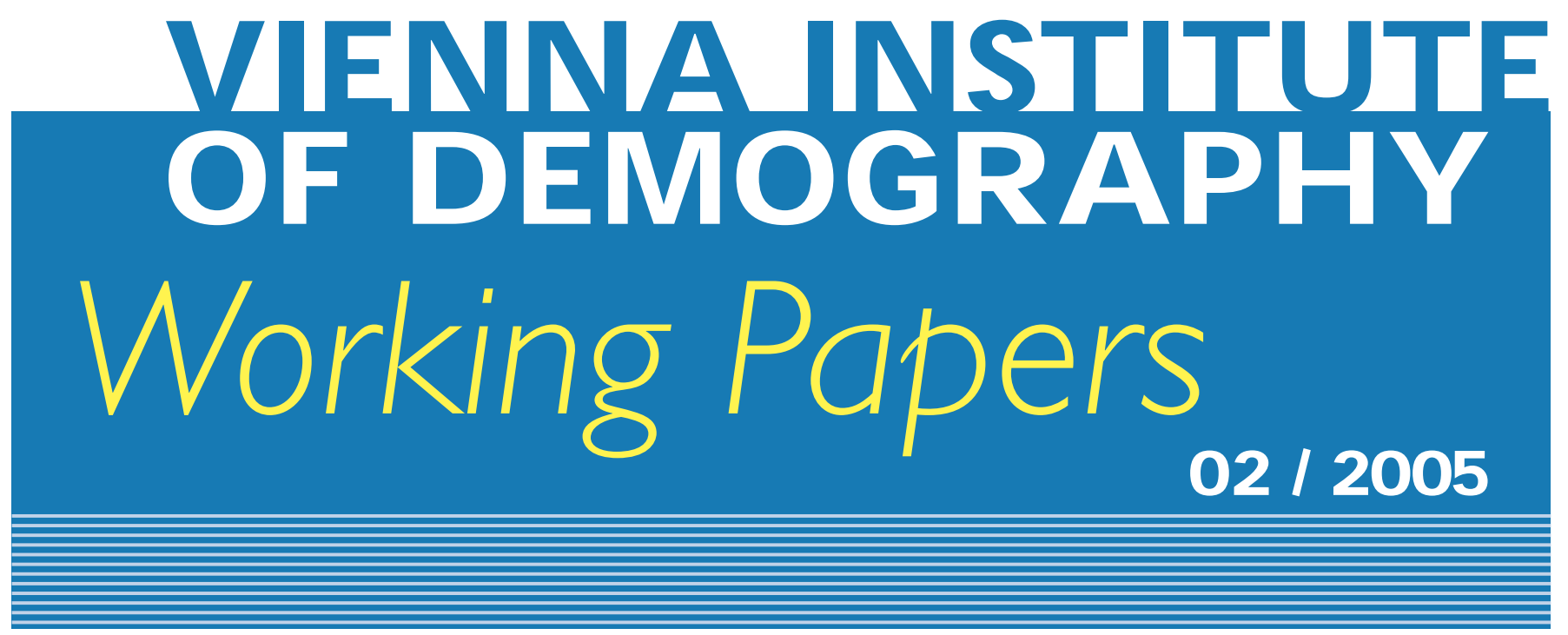

Engelhardt, Henriette and Alexia Prskawetz

\title{
Arbeitsmarkt und Demographie
}

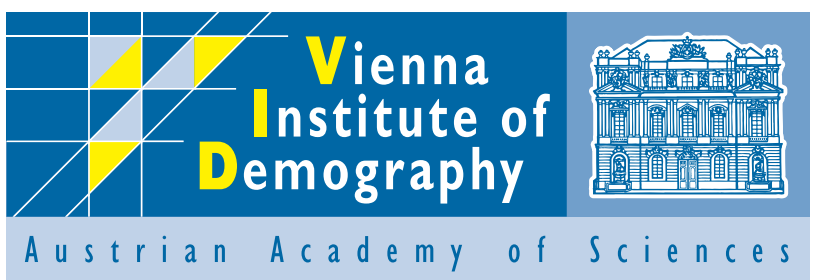

Vienna Institute of D emography

A ustrian A cademy of Sciences

Prinz Eugen-Strasse 8-10 · A-1040 Vienna · A ustria

E-Mail: vid@ oeaw.ac.at

W ebsite: www.oeaw.ac.at/vid 


\section{Zusammenfassung}

Im vorliegenden Beitrag werden die wechselseitigen Beziehungen von demographischen Veränderungen und Arbeitsmarktentwicklungen anhand eines Literaturüberblicks diskutiert. Insbesondere werden die Effekte von demographischen Strukturen und Prozessen auf die Lohnstruktur und auf die Arbeitslosigkeit präsentiert, wobei sowohl Mikro- als auch Makroansätze diskutiert und die Implikationen der Bevölkerungsalterung für den Arbeitsmarkt besprochen werden. In Mikroansätzen werden z.B. altersspezifische Profile der Humankapitalbildung als optimale Lösung eines individuellen Entscheidungsproblems modelliert. Im Gegensatz dazu werden in Makroansätzen die Beziehung von Makrovariablen, z.B. Jugendarbeitslosigkeit und Besetzungsstärke der eigenen Kohorte, modelliert. Schließlich stellen wir verschiedene theoretische Perspektiven vor, welche Effekte arbeitsmarktrelevanter Faktoren auf die Fertilität untersuchen.

\section{Schlüsselwörter}

Bevölkerungsalterung, Fertilität, Kohortenstärke, Arbeitslosigkeit, Lohn- und Beschäftigungsstruktur

\section{Danksagung}

Für Anregungen, Hinweise und Kommentare danken wir Martin Abraham, Thomas Hinz, Vegard Skirbekk und Maria Winkler-Dworak.

\section{Autoren}

Henriette Engelhardt ist wissenschaftliche Mitarbeiterin am Institut für Demographie.

Alexia Prskawetz ist stellvertretende Direktorin und Leiterin der Forschungsgruppe „Bevölkerungsökonomie“" am Institut für Demographie. 


\title{
Arbeitsmarkt und Demographie
}

\author{
Henriette Engelhardt und Alexia Prskawetz
}

\section{Problemstellung}

Seit einigen Jahrzehnten ist die Bevölkerungsentwicklung in den meisten Industrieländern durch niedrige Fertilität und sinkende Mortalität gekennzeichnet. Die daraus resultierende Bevölkerungsalterung führte zu einem verstärkten Interesse an den sozialen, ökonomischen und politischen Konsequenzen, welche aus den sich ändernden demographischen Prozessen und Strukturen zu erwarten sind. Während oft die Nachhaltigkeit der Sozialsysteme (Gesundheits-, Pensions- und Pflegesysteme) im Vordergrund steht, beschäftigen sich zahlreiche neuere Studien mit den Auswirkungen der Demographie auf den Arbeitsmarkt und hier insbesondere auf die Beschäftigung, die Arbeitslosigkeit und die Lohnstruktur (vgl. Johnson und Zimmermann 1993; Herfurth et al. 2003; Börsch-Supan 2002).

Neben diesem durch die Bevölkerungsalterung hervorgerufenen Interesse an demographischen Erklärungsansätzen für die Entwicklung von Arbeitsmarkindikatoren existiert eine umfangreiche Literatur zu Veränderungen in der Entwicklung und Zusammensetzung der Bevölkerung und deren Auswirkungen für die Angebotsseite des Arbeitsmarktes (vgl. Blien und Meyer 2000). Auf der Mikroebene wird dabei der Zusammenhang zwischen dem individuellen Alter und Löhnen, Ausbildung, Erwerbsquoten, Arbeitslosigkeit und Beschäftigungsverhältnissen untersucht. Zusätzlich sind das Geschlecht und die Nationalität weitere wichtige demographische Variablen, nach welchen Arbeitsmarktindikatoren häufig differieren. Auf der Makroebene belegen zahlreiche Studien einen (wenn auch nicht eindeutigen) Zusammenhang zwischen Löhnen und Erwerbsquoten verschiedener Generationen und deren Besetzungsstärke, d.h. der Kohortengröße. Die Motivation dieser Studien bildeten die schlechten Arbeitsmarktbedingungen, welche die Babyboomgeneration in den USA bei ihrem Eintritt in den Arbeitsmarkt in den 70er Jahren des letzten Jahrhunderts vorfand.

Demographische Veränderungen haben nicht nur Auswirkungen auf den Arbeitsmarkt, sondern zahlreiche Studien belegen einen Effekt arbeitsmarktrelevanter Faktoren auf die Fertilitäts- und Migrationsentscheidung und vereinzelt auch auf die Mortalitätsentwicklung. Eine der am häufigsten analysierten Fragestellungen in diesem Zusammenhang ist die Beziehung von Frauenerwerbstätigkeit und Fertilität. Während die jährliche Korrelation zwischen der Frauenerwerbsquote und der Gesamtfertilitätsrate ${ }^{1}$ in 20 OECDLändern Mitte der 1980er Jahre einen Wechsel von einem negativen zu einem positiven Wert zeigt (vgl. Abbildung 1), belegen jüngste Studien (Engelhardt et al. 2004, Engelhardt und Prskawetz 2004, 2005) anhand von Zeitreihenmodellen für einzelne Länder bzw. Panelmodellen für zusammengefasste Zeitreihen von Quer- und Längsschnittsdaten, dass die negative Beziehung von Frauenerwerbstätigkeit und Fertilität über die Zeit erhalten bleibt bzw. abgeschwächt wird, nicht jedoch ihr Vorzeichen wechselt.

\footnotetext{
${ }^{1}$ Die Gesamtfertilitätsrate gibt die nach Alter standardisierte Anzahl Geburten je 1000 Frauen im gebärähigen Alter zwischen 14 und 49 Jahren an.
} 


\section{Abbildung 1}

Korrelation zwischen Fertilität und Frauenerwerbstätigkeit für 21 OECD-Länder, 19602000

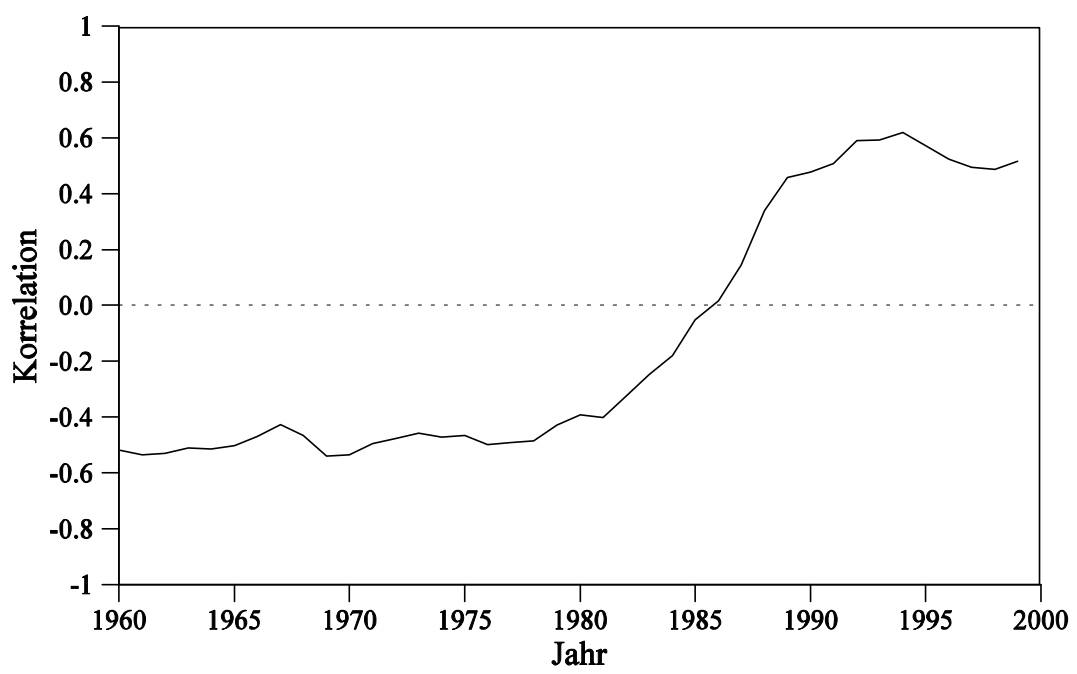

Im vorliegenden Beitrag werden die wechselseitigen Beziehungen von demographischen Veränderungen und Arbeitsmarktentwicklungen diskutiert. In Kapitel 2 werden zunächst die Effekte von demographischen Strukturen und Prozessen auf die Lohnstruktur und auf Arbeitslosigkeit präsentiert. Dabei werden sowohl Mikro- als auch Makroansätze diskutiert und die Implikationen der Bevölkerungsalterung für den Arbeitsmarkt besprochen. In Mikroansätzen werden z.B. altersspezifische Profile der Humankapitalbildung als optimale Lösung eines individuellen Entscheidungsproblems modelliert. Im Gegensatz dazu werden in Makroansätzen die Beziehung von Makrovariablen, z.B. Jugendarbeitslosigkeit und Besetzungsstärke der eigenen Kohorte, modelliert. In Kapitel 3 stellen wir unterschiedliche Modelle vor, welche Effekte arbeitsmarktrelevanter Faktoren auf die Fertilität untersuchen. Die Arbeit endet mit einem Resümee des bisherigen Standes der Forschung und einem Ausblick auf weiteren Forschungsbedarf.

Unser Ziel ist es nicht, einen vollständigen Überblick, sondern einen Einblick in ausgewählte, aktuelle Themen der Wechselbeziehung von Demographie und Arbeitsmarkt zu geben. Wir haben dabei die Fertilität als ein zentrales demographisches Phänomen und die Besetzungsstärke von Geburtskohorten als eine zentrale demographische Strukturvariable gewählt, welche in wechselseitiger Beziehung zu Lohn- und Beschäftigungsstruktur steht. 


\section{Demographische Veränderungen und Arbeitsmarktindikatoren}

\subsection{Altersstruktur und Lohnstruktur auf der Mikroebene}

Der Zusammenhang zwischen Alter und Ausbildung, Berufserfahrung, Seniorität und Löhne wurde in zahlreichen Arbeiten untersucht, die vor allem den Arbeiten von Schultz (1961), Becker (1962) und Mincer (1958, 1962, 1970, 1974) folgen. (Die folgenden Ausführungen basieren auf der Arbeit von Klevmarken 2003a.) Die Humankapitaltheorie liefert das theoretische Fundament für die Herleitung der altersspezifischen Lohnkurve. Die wesentliche Annahme ist hierbei, dass die individuelle Entscheidung, in Humankapital zu investieren (durch Schulausbildung und on-the-job training), durch das zu erwartende zukünftige Einkommen bestimmt wird. Theoriegemäß wird man nur dann in Humankapital investieren, wenn die dadurch bedingten Einkommensverluste durch zukünftige Einkommenssteigerungen ausgeglichen werden. Als Resultat dieser Mikrofundierung ergibt sich eine mit der Berufserfahrung (und indirekt somit mit dem Alter) zunächst steigende und dann fallende Lohnkurve. Das Maximum der Lohnkurve wird dabei für verschiedene Karriereverläufe in unterschiedlichen Altersgruppen angenommen.

Die zentrale Einkommensgleichung von Mincer (1974) modelliert das logarithmierte Einkommen als Funktion der Schuljahre, der Jahre der Berufserfahrung und des Quadrats der Jahre der Berufserfahrung. Erweiterungen der Mincer-Gleichung beinhalten oft jedoch auch die Altersvariable als zusätzlichen erklärenden Faktor, durch den die Abschreibung des Humankapitals, also das „Veralten von Wissen und Fähigkeiten“, modelliert wird. Empirisch ist es jedoch schwierig, zwischen einem internen Abschreibungsfaktor durch physische Alterung und einem externen, Vintage'-Effekt der Abschreibung (d.h. das Humankapital älterer Erwerbstätiger entspricht oft nicht mehr den aktuellen Anforderungen am Arbeitsmarkt) zu unterscheiden. In diesem Zusammenhang sind auch die Untersuchungen zur Lohnstruktur von Frauen mit Kindern, welche ihren Beruf unterbrochen haben, anschlussfähig (Mincer und Polacheck 1974, Mincer und Ofek 1982, Gustafsson 1981, Budig und England 2001). Die diesbezügliche empirische Evidenz ist jedoch nicht eindeutig, ein negativer Effekt der Berufsunterbrechung auf die Lohnprofile kann also nicht mit Sicherheit belegt werden. Des Weiteren haben zahlreiche empirische Studien gezeigt, dass die altersspezifische Lohnkurve vom Schul- und Berufstyp abhängt und diese Ergebnisse für verschiedene Länder unterschiedlich sind (Lillard und Weiss 1979, Gustafsson 1976). Zum Beispiel zeigte Klevmarken (1982), dass die Alters-Lohnprofile für Arbeiter mit geringer Schulausbildung in Schweden flacher sind als für die entsprechenden Gruppen von Arbeitnehmern in Amerika. Für Arbeitnehmer mit höherer Schulausbildung (Mittelschule, Universität) sind diese Unterschiede geringer. In vielen Studien konnten auch geschlechtsspezifische Unterschiede in der altersspezifischen Lohnstruktur unabhängig von den bereits erwähnten Erwerbsunterbrechungen nachgewiesen werden, wobei Frauen gegenüber Männern eine geringere und flachere Lohnkurve aufweisen. Wie weit dieser Effekt auf Diskriminierung oder auf unbeobachtete Heterogenität zurückzuführen ist, kann nicht eindeutig festgestellt werden (Reskin und Bielby 2005; Jann und Engelhardt 2005)

Neben dieser Mikrofundierung ist der Makroeffekt einer sich ändernden Altersverteilung auf die Lohnstruktur von großer Bedeutung. 


\subsection{Kohorteneffekte}

\subsubsection{Kohorteneffekte und Lohnprofile}

Während in der Humankapitaltheorie Löhne als Funktion der individuellen Bildungsund Berufserfahrungsjahre sowie anderer Kontrollvariablen modelliert werden, wird in der Literatur ,konkurrierender Generationen auf dem Arbeitsmarkt' davon ausgegangen, dass die relative Größe der eigenen (und eventuell benachbarter) Kohorten einen Effekt auf die Höhe und Form der altersspezifischen Lohnstruktur hat (Welch 1979, Easterlin 1987). Die Annahme ist hierbei, dass ältere und jüngere Arbeitskräfte am Arbeitsmarkt nicht perfekt substituierbar sind. Eine stärker besetzte jüngere Kohorte wird somit am Arbeitsmarkt mit sich selbst im Wettbewerb stehen, die relativen Löhne der jüngeren zu den älteren Arbeitskräften werden als Folge sinken. Entsprechend der These von Easterlin wird dieser Kohorteneffekt über den gesamten Lebenszyklus gültig sein.

Stapeltone und Young (1988) argumentieren, dass Individuen auf ihre Position im demographischen Zyklus reagieren und unterschiedliche Humankapitalinvestitionen tätigen. Diesen Überlegungen liegt die Annahme zugrunde, dass der Babyboom der Nachkriegsgeneration zu starken Fluktuationen in den individuellen Erträgen der Bildungsinvestionen geführt hat. Unter der Annahme, dass die Substituierbarkeit zwischen jüngeren und älteren Arbeitnehmern mit wachsender Bildung sinkt, wird in einer stärker besetzten Kohorte das Lebenseinkommen für höher Gebildete stärker reduziert wodurch wiederum die Anreize der Humankapitalinvestition fallen. Dieser Theorie entsprechend sind die Einstiegsgehälter für stärker besetzte Kohorten größer, während die Lohnkurve über die Zeit im Vergleich zu benachbarten, schwächer besetzten Kohorten geringer ist.

Neben der absoluten Größe einer Kohorte ist die Position innerhalb eines demographischen Zyklus ebenso von Bedeutung. In der Theorie wird argumentiert, dass Kohorten welche im Aufschwung eines demographischen Zyklus geboren sind ökonomisch besser gestellt sind als Kohorten welche im Abschwung geboren sind. Als Erklärung wird die These von Easterlin (1987) angewandt, wonach die Substituierbarkeit von Arbeitern verschiedenen Alters die Einkommenssituation der Individuen wiederspiegelt. Da Arbeitnehmer welche im Abschwung eines demographischen Zyklus geboren sind noch mit den stark besetzten Kohorten des Babyboom am Arbeitsmarkt konkurrieren, wird deren Einkommen geringer sein.

Die empirische Evidenz zu Kohorteneffekten auf Lohnprofile ist jedoch nicht eindeutig. Welch (1979) findet für US- amerikanische Daten einen negativen Einkommenseffekt für stärker besetzte Kohorten und zeigt, dass dieser negative Effekt für höhere Bildungsgruppen stärker ist. Als mögliche Erklärung für letzteres Ergebnis gibt er die höhere Substituierbarkeit zwischen Arbeitskräften in niedrigeren Bildungsgruppen an. Im Gegensatz zu Welch - und entsprechend der Theorie des erweiterten Humankapitalansatzes von Stapeltone und Young - findet Berger (1989) einen positiven Effekt der eigenen Kohortengröße auf die Höhe der Einstiegslöhne, jedoch einen negativen Effekt auf die Erträge der bereits akkumulierten Berufserfahrung. Ein Anstieg der Besetzungsstärke benachbarter Kohorten führt hingegen zu geringeren Einstiegslöhnen, aber einem größeren Anstieg der Lohnkurve über die Zeit. In Summe führt dies zu flacheren Lohnprofilen für stärker besetz- 
te Kohorten. Während die meisten Studien auf US-amerikanischen Daten beruhen, gibt es nur wenige Untersuchungen für europäische Länder. Hier sei vor allem die Arbeit von Klevmarken (1993b) erwähnt, welche anhand schwedischer Daten keinen derartigen Kohorteneffekt fand, oder die komparativen Untersuchungen für 16 europäische Länder von Wright (1989), in der ebenfalls keine Kohorteneffekte auf die Lohnprofile gezeigt werden konnten. Im Gegensatz dazu konnten Ermisch (1988) und Wright (1991) für britische Daten einen negativen Effekt der Kohortengröße auf Löhne nachweisen. Analog zu Berger (1989), jedoch unter Verwendung eines Paneldatensatezs für Schweden für die Jahre 1968 bis 1999, zeigen Dahlberg und Nahum (2003), dass stärker besetzte Kohorten ein im Vergleich zu schwächer besetzten Kohorten höheres Einkommen haben, die Erträge in Bezug auf bereits akkumulierte Berufserfahrung jedoch für unterschiedliche Bildungsgruppen ungleich sind. Ihre Ergebnisse zeigen einen flacheren Anstieg des Lohnprofils für Arbeiter höherer Bildungsgruppen im Vergleich zu Arbeitern niedrigerer Bildungsgruppen.

Die These, dass die Position innerhalb des demographischen Zyklus von Bedeutung ist, wird von Macunovich (1999) aufgegriffen. In ihrer empirischen Studie anhand aktualisierter Daten von Welch (1979) und Berger (1989) zeigt sie, dass Kohorten, welche einem Babyboom folgen, ökonomisch schlechter gestellt sind als Kohorten, welche dem Babyboom vorangehen. Die Ergebnisse in Berger (1989) entsprechen diesen Resultaten: Wer im Abstieg eines demographischen Zyklus geboren wurde, ist ökonomisch schlechter gestellt als wer im Aufschwung eines demographischen Zyklus geboren wird.

\subsubsection{Kohorteneffekte und Arbeitslosigkeit}

Eine verwandte Literatur untersucht die Effekte der Kohortengröße auf die Arbeitslosigkeit. Entsprechend der neoklassischen Wachstumstheorie führt ein Anstieg des Faktors Arbeit zu einer geringeren Kapitalintensität pro Arbeiter und als Folge zu einem höheren Zinssatz und einem geringeren Lohn. Unter der Annahme friktioneller Arbeitsmärkte kann der niedrigere Lohn dann zu einer höheren Arbeitslosigkeit führen, wenn arbeitslose Arbeitskräfte ihre Anstrengungen der Arbeitsplatzsuche reduzieren. Die unterschiedliche Besetzungsstärke einzelner Kohorten kann somit die altersspezifischen Arbeitslosigkeitsraten verändern und indirekt damit die aggregierte Arbeitslosigkeit. Neben diesem indirekten Effekt, wird die aggregierte Arbeitslosigkeit, welche ein gewichteter Durchschnitt der Arbeitslosigkeit von Arbeitern einzelner Altersgruppen ist, auch direkt über die Änderung der Altersstruktur verändert da diese die Gewichte der Aggregation verändern wird.

Das Problem besteht nun in der Messung des indirekten Effekts, wobei ökonometrische Analysen meist auf Zeitreihenbeobachtungen beruhen. Eine oft zitierte Kritik basiert auf der Tatsache, dass makroökonomische Veränderungen wie die sinkende Nachfrage nach jungen Arbeitern als Folge des wirtschafltichen Abschwungs und technologischer Veränderungen und nicht die zu messende demographische „Tatsache“ wie der Babyboom (d.h. stärker besetzte Kohorten) für die höhere Arbeitslosigkeit der Babyboomgeneration verantwortlich waren. Um dieser Kritik zu begegnen, haben Korenman und Neumark (1997) ein Panel von 15 Ländern über 20 Jahre heran gezogen. Eine solche Paneldatenanalyse erlaubt die internationale Variation in der Größenordnung und der zeitlichen Abfolge der Änderungen in der Kohortengröße junger Arbeitnehmer zu nützen, um so die Kohor- 
teneffekte besser von Periodeneffekten welche alle Jugendlichen in gleichem Ausmaß betroffen haben (z.B. wachsende Nachfrage nach qualifizierten Arbeitskräften), zu isolieren. Die Ergebnisse von Korenman und Neumark zeigen einen signifikanten, positiven Effekt der Kohortengröße auf die Jugendarbeitslosenrate im Vergleich zur Arbeitslosenrate der Erwerbsbevölkerung im mittleren Alter, wobei dieser Effekt umso stärker ist, je geringer die Flexibilität am Arbeitsmarkt ist. Bloom et al. (1987) haben ebenso ein Panel von Zeitreihen und Querschnittsdaten untersucht und konnten zeigen, dass stärker besetzte Kohorten in den USA niedrigere Löhne erhielten, während in Europa bei jüngeren und stärker besetzten Kohorten die Jugendarbeitslosigkeit anstieg. Dies zeigt, dass institutionelle Rahmenbedingungen des Arbeitsmarktes die Auswirkungen demographischer Veränderungen wesentlich beeinflussen. Ein nicht zu unterschätzender Faktor ist natürlich auch, dass der Arbeitsmarkt für jüngere Arbeiter wesentlich sensitiver auf ökonomische Entwicklungen reagiert (z.B. die wachsende Nachfrage nach qualifizierten Arbeitnehmern, vgl. Korenman und Neumark 1997).

In einer bahnbrechenden Arbeit weist Shimer (2001) auf zwei wesentliche Schwachpunkte in den bisherigen empirischen Studien hin. Einerseits werden makroökonomische Veränderungen, welche die Arbeitslosenraten unabhängig von der Besetzungsstärke der Kohorten beeinflussen, ignoriert. Eine zweite Kritik befasst sich mit der Substituierbarkeit von Arbeitnehmern unterschiedlichen Alters. Falls Arbeiter jungen und mittleren Alters in der Produktivität komplementär sind, hätte der Babyboom eventuell die Arbeitslosigkeit der Erwerbsbevölkerung mittleren Alters reduziert. In diesem Falle würden die indirekten demographischen Effekte die direkten Effekte kompensieren. Die Studie von Korenman und Neumark stellt sich der ersten Kritik, da sie Zeitreihendaten für 15 Länder analysiert und somit für makroökonomische Zeiteffekte kontrollieren kann. Die Tatsache, dass sie die Arbeitslosigkeit junger Arbeitnehmer im Verhältnis zur Arbeitslosigkeit der Arbeitnehmer mittleren Alters untersuchen macht sie jedoch bezüglich der zweiten Kritik angreifbar. Bei einem Anstieg dieses Verhältnisses kann nicht identifiziert werden, ob es zu einer Erhöhung der Arbeitslosigkeit junger Arbeitnehmer oder zu einer Reduktion der Arbeitslosigkeit der Arbeitnehmer mittleren Alters gekommen ist. Die Innovation der Arbeit von Shimer ist die Fokussierung auf Daten innerhalb der USA und hier insbesondere jährlicher Arbeitslosenraten für jeden Bundesstaat. Innovativ sind sowohl der relative große Datensatz, als auch die Tatsache, dass durch ein Zeitreihenpanel innerhalb eines Staates die zeitlichen Änderungen in makroökonomischen Größen besser erfassbar ist (im Vergleich zu einem Zeitreihenpanel über verschiedene Länder mit unterschiedlichen makroökonomischen Entwicklungen). Im Gegensatz zu den bisherigen Arbeiten fand Shimer, dass ein Anstieg des Anteils junger Arbeitskräfte die Jugendarbeitslosigkeit senkt . Shimer präsentiert und testet außerdem ein theoretisches Modell, welches diesen ökonometrischen Studien zugrunde liegt, wobei er von der Angebotsseite am Arbeitsmarkt ausgeht. Da jüngere Arbeitskräfte mobiler sind und eher ein Jobangebot annehmen, ist es für Firmen optimal, in Staaten mit jüngerem Arbeitskräftepotential Arbeitsplätze zu schaffen. Die Ergebnisse können somit dadurch erklärt werden, dass Bundesstaaten mit höherem Anteil an jungen Arbeitnehmern, die Ansiedlung von Firmen und daher die Schaffung von Arbeitsplätzen positiv beeinflussen.

Für Deutschland hat Zimmermann (1991) anhand westdeutscher Daten keinen negativen Effekt der Kohortengröße auf die Arbeitslosigkeit der jungen Arbeitnehmer nachwei- 
sen können. $\mathrm{Zu}$ einem analogen Ergebnis kommt auch Schmidt (1993) für Westdeutschland vor 1990. Nur für die Altersgruppe der 55- bis 59-jährigen Männer und Frauen und die Altersgruppe der 25- bis 34-jährigen Männer konnte er eine positive Beziehung zwischen deren Kohortengröße und ihrer Arbeitslosigkeit finden.

Eine ausführliche Diskussion zu demographisch bedingter Arbeitslosigkeit findet sich ebenso in Blien und Meyer (2000: 1025 ff.). Neben den neoklassischen und keynesianischen Ansätzen werden hier insbesondere die Effizienzlohn- und Verhandlungsansätze diskutiert, welche dem europäischen Arbeitsmarktmodell entsprechen (vgl. Hinz und Abraham, Kapitel 2). In den letzteren Modellen sind die Löhne flexibel und sinken nicht auf ein markträumendes Niveau wie im neoklassischen Modell, da dies durch die Firmen oder die Gewerkschaften verhindert wird. Generell kann die Arbeitslosigkeit nicht dem Auftreten bestimmter Gruppen (Frauen, Ausländer) zugeschrieben werden, sondern es werden nur Teilarbeitsmärkte davon betroffen, d.h. jene, mit welchen die betrachtete Gruppe von Arbeitnehmern konkurriert.

Abschließend sei noch auf die Überblickstabellen zu Studien zum Zusammenhang von Kohortengröße und Arbeitslosigkeit bzw. Kohortengröße und Löhnen in Bloom et al. (1987, Tabelle 3) und Korenmark und Neumark (1997, Tabelle 1) hingewiesen. Die allgemeine empirische Evidenz lässt für zahlreiche Länder auf einen positiven Effekt der Kohortengröße auf die Jugendarbeitslosigkeit und einen negativen Effekt auf Beschäftigung und Löhne schließen, wobei diese Effekte vom Ausbildungsniveau abhängen. Generell kann davon ausgegangen werden, dass Arbeitnehmer verschiedenen Alters keine perfekten Substitute sind und Arbeitnehmer mit niedriger Qualifikation leichter über die Altersgruppen hinweg ersetzbar sind. In den meisten Studien kann ein Aufholeffekt über den Lebenszyklus nachgewiesen werden (d.h. Jahrgänge mit niedrigeren Einstiegsgehältern holen ihren anfänglichen Einkommensnachteil über den Lebenszyklus auf). Kohorteneffekte werden ebenso von der Position der entsprechenden Generation im demographischen Zyklus abhängen und in der Regel für besser ausgebildete Arbeitskräfte stärker sein

\subsection{Konsequenzen der alternden Bevölkerung für den Arbeitsmarkt}

Zentrales Thema in der Diskussion über Bevölkerungsentwicklung und Arbeitsmarkt sind in den letzten Jahren die Auswirkungen einer alternden Bevölkerung auf das Arbeitsangebot, welches nicht nur quantitativ abnimmt, sondern auch älter wird (vgl. Herfurth et al. 2003 sowie Arnds und Bonin 2003). Die Alterung der Arbeitskräfte wird in Deutschland früher einsetzen als die Alterung der Gesamtbevölkerung. Entsprechend Arnds und Bonin (2003: 134) ist bei Fehlen einer substanziellen Zuwanderung bereits ab 2015 mit einem geringeren und älteren Arbeitsangebot zu rechnen. Die Argumentation, dass das zu erwartende geringere Arbeitsangebot zu einer geringeren Arbeitslosigkeit führen wird, ist jedoch für Deutschland sehr fragwürdig, da die Arbeitslosigkeit in Deutschland neben konjunkturellen vor allem friktionelle (geringe berufliche und räumliche Mobilität) und strukturelle (Fehlanreize im Sozialsystem) Ursachen hat. 
Im Folgenden orientieren wir uns an dem Übersichtsaufsatz von Arnds und Bonin (2003) und stellen die Wirkungen eines alternden Arbeitsangebots auf die Arbeitsproduktivität, die Entlohnung und die Humankapitalbildung vor.

Allgemein besteht über den Umfang und die Struktur der künftigen Erwerbsbevölkerung große Unsicherheit. Während sich demographische Entwicklungen mittelfristig relativ gut vorhersagen lassen, wird die Erwerbsbevölkerung von den Erwerbsquoten, den Jahres- und Lebensarbeitszeiten und der Rekrutierung stiller Reserven (z.B. Frauen, älterer Arbeitnehmer etc.) beeinflusst. Diese Determinanten können wiederum durch demographische Veränderungen beeinflusst werden. Modellrechnungen von Zimmermann (1991, zitiert in Arnds und Bonin 2003) zeigen, dass bei hoher Erwerbsquote die 40-MillionenGrenze der Erwerbsbevölkerung erst um das Jahr 2020 unterschritten wird. Bei einer pessimistischen Variante mit niedriger Zunahme der Erwerbsneigung ist mit einem Unterschreiten der 40-Millionen-Grenze bereits um $2010 \mathrm{zu}$ rechnen. Unabhängig von den zukünftigen Erwerbsquoten wird sich jedoch von 2020 an ein durch die Geburtenausfälle der Vergangenheit ausgelöster absoluter Rückgang der Erwerbsbevölkerung vollziehen. Als Folge der Reduktion des Erwerbspotenzials könnte laut Börsch-Supan (2001) das Bruttosozialprodukt pro Kopf zwischen 2010 und 2035 um 15 Prozent sinken. Eine höhere Kapitalintensität als Folge des schrumpfenden Erwerbspotentials könnte diesen Wohlstandsverlust zur Hälfte ausgleichen (vgl. Börsch-Supan 2001). Um den Wohlstand beizubehalten, ist somit eine Erhöhung der Arbeitsproduktivität oder wachsender technologischer Fortschritt erforderlich. Diese notwendigen ökonomischen Entwicklungen könnten jedoch in Zukunft noch schwieriger werden. Mit dem Alter nehmen gesundheitliche Probleme zu und der rasche technologische Fortschritt erfordert Weiterbildungsaktivitäten bis ins hohe Alter, die bislang nicht gegeben sind. Nur teilweise kann die altersbedingte Leistungsbeschränkung durch die altersbedingte Zunahme an Berufserfahrungen kompensiert werden (vgl. Baltes und Baltes 1989, Salthouse 1991).

Zusammenfassend kann festgestellt werden, dass die Effekte der Bevölkerungsalterung auf die Arbeitsproduktivität nicht zu belegen sind. So können biomedizinische Forschungen die These einer allgemeinen Leistungsminderung älterer Arbeitnehmer nicht bestätigen. Ein weiteres Argument, welches gegen eine eindeutige Beziehung zwischen Alter und Arbeitsproduktivität spricht, ist die Tatsache, dass sich die effektive Produktivität älterer Arbeitnehmer erst durch das Zusammenwirken mit innerbetrieblichen Organisationsabläufen und Produktionsstrukturen ergibt. So können betriebliche Reorganisationen zu einer Produktivitätsminderung mit dem Alter führen, während produktivitätssteigernde Entwicklungen mit dem Alter ebenso möglich sind (z.B. steigt die Qualität der, job matches' mit dem Alter). Da individuelle Produktivität schwierig zu messen ist, sind zuverlässige empirische Aussagen über den Zusammenhang von Alter und Produktivität schwer möglich (vgl. Skirbekk 2004).

Unter der Annahme vollkommener Arbeitsmärkte wäre die Auswirkung einer demographischen Alterung auf die Entlohnung der Arbeitskräfte ein Anstieg des Preises der Arbeitskraft, da dieser Faktor im Vergleich zu Kapital relativ knapper wird. Wie bereits in Kapitel 2 von Hinz und Abraham diskutiert, ist der Arbeitsmarkt in Bezug auf Information und Präferenzen unvollkommen, so dass die Entlohnung nicht notwendigerweise den Knappheitsrelationen entspricht und die Löhne nicht mit der altersspezifischen Produktivi- 
tät übereinstimmen. ,Job matching'-Modelle und ,Shirking'-Modelle lösten in den letzten Jahren das neoklassische Modell der Arbeitsnachfrage ab. Die Folge dieser neuen lohntheoretischen Überlegungen ist ein altersspezifisches Lohnprofil, bei dem Arbeitskräfte am Beginn ihres Erwerbslebens einen unter ihrer Produktivität liegenden Lohnsatz erhalten und in höheren Altersjahren sich diese Relation umkehrt. Im theoretischen Idealfall ist der Wert der erbrachten Produktivität gleich dem Wert des Einkommensstroms über die gesamte Verweildauer im Betrieb. Diese über den Lebenszyklus gültige Übereinstimmung von Produktivitäts- und Einkommensprofil wird jedoch nicht auf Betriebsebene in einer Querschnittsanalyse zu beobachten sein. Die Altersstruktur der Arbeitnehmer auf Betriebsebene entspricht notwendigerweise nicht jener Altersstruktur für welche das Periodeneinkommen gleich dem Periodenproduktivitätsprofil ist. Insbesondere wird bei einem steigenden Anteil älterer Arbeitnehmer die Differenz zwischen betrieblichen Lohnkosten und den Erträgen aus Einsatz der Arbeitskraft ansteigen. Um diesem Trend entgegenzuwirken, werden Betriebe die Alters-Lohn-Profile anpassen, wobei sowohl geringere Anfangsgehälter als auch die Alternative einer Abflachung des Lohnanstiegs mögliche Entwicklungen darstellen. Geringere Anfangsgehälter aufeinanderfolgender Kohorten wurden von Luckert et al. (1995) nachgewiesen.

Neben diesen durch die demographische Altersverschiebung ausgelösten Lohnanpassungen kann es auch zu Niveaueffekten auf die Entlohnung des Produktionsfaktors Arbeit kommen. So sollte die Knappheit des Faktors Arbeit die Löhne positiv beeinflussen. Da der Faktor Arbeit jedoch heterogen ist, werden die Lohnauswirkungen einer demographischen Alterung auf Teilarbeitsmärkte von der relativen Knappheitsrelation der Arbeitskräfte in den einzelnen Teilarbeitsmärkten bestimmt. Wie bereits in Abschnitt 2.2. diskutiert, wird hierbei die Substituierbarkeit von Arbeitskräften verschiedenen Alters eine wesentliche Rolle spielen.

Die Auswirkungen demographischer Alterung auf das gesamtwirtschaftliche Humankapital sind durch exogene Bestandsveränderungen und endogene Verhaltensänderungen bezüglich der Humankapitalinvestitionen zu erklären. Bestandsveränderungen ergeben sich als Resultat der unterschiedlichen kohortenspezifischen Humankapitalausstattung. Sind laut Arnds und Bonin (2004: 151) ,unter den heute 35- bis 45-Jährigen weniger als ein Fünftel ohne Berufsabschluss, (so) sind es bei den älteren Jahrgängen über 30 Prozent". Da bildungsschwache Jahrgänge zunehmend in den Ruhestand treten, könnte dies der alterungsbedingten Verschlechterung der gesamtwirtschaftlichen Arbeitsproduktivität entgegenwirken. Andererseits werden die Renditen bezüglich der Humankapitalbildung mit zunehmendem Angebot an Humankapital zurückgehen; dies könnte zu den erwähnten endogenen Verhaltensänderungen auf Individualebene führen. Ein Zusammenhang zwischen demographischer Entwicklung und Ausbildungsrendite konnte allerdings für Deutschland jedoch bisher nicht nachgewiesen werden (vgl. Arnds und Bonin 2003: 155ff). Der demographische Schrumpfungsprozess könnte überdies zu einem Anstieg der Humankapitalrendite für die geburtenschwachen Erwerbsjahrgänge führen und dadurch der quantitativen Reduktion der Erwerbsbevölkerung entgegenwirken. Weiter führend sind die Überlegungen von Arnds und Bonin, denen zu Folge der Anstieg der Humankapitalrendite auch eine Investition in Humankapital bei kürzerem Erwerbshorizont lohnend macht (wie dies vor allem für ältere Arbeitnehmer der Fall ist). 
Im vorangegangenen Kapitel haben wir einen Überblick über die Auswirkungen demographischer Entwicklungen (und hier insbesondere der Änderungen der Kohortengrößen und der Bevölkerungsalterung) auf arbeitsmarktrelevante Faktoren wie Löhne, Arbeitslosigkeit und Humankapitalinvestition gegeben. Natürlich kann hier kein vollständiger Überblick erfolgen, da wir beispielsweise die Auswirkungen von Migrationen und Mortalitätsveränderungen auf den Arbeitsmarkt ausgeklammert haben. Wir werden uns daher auch im zweiten Teil unseres Beitrags auf ein demographisches Phänomen, die Fertilität, beschränken und einige der theoretischen Modelle vorstellen, welche die Auswirkungen von arbeitsmarktrelevanten Faktoren auf die Fertilitätsentwicklung untersuchen.

\section{Die Rolle des Arbeitsmarktes für die Fertilität}

Die zwei bekanntesten Schulen zur Erklärung der Beziehung zwischen Fertilität und Frauenerwerbstätigkeit sind der familienökonomische Ansatz und das Easterlin-Modell der relativen Einkommenshypothese. Beiden Theorien gemeinsam ist das Ziel, die negative Beziehung zwischen Fertilität und Frauenerwerbstätigkeit zu erklären. Die familienökonomische Theorie stellt die Rolle der Frauenlöhne als Opportunitätskosten der Kindererziehung in den Vordergrund (z.B. das Modell von Willis 1973 und seine Anwendung von Butz und Ward 1979) und betrachtet primär Änderungen des Wertes der Zeit, während Easterlin $(1980,1987)$ - wie oben bereits angedeutet - auf die Änderungen des relativen Einkommens durch demographische Zyklen abstellt (,baby boom' und ,baby bust').

Sind die die Ansätze der Neuen Haushaltsökonomik und der relativen Einkommenseffekte (Easterlin) primär statisch angelegt sind, berücksichtigen Lebenslauftheorien explizit, dass Familienentwicklung und Erwerbstätigkeit ein Teilprozess des Lebensverlaufs von Menschen sind, den Menschen planen und gestalten. Im Folgenden diskutieren wir diese drei Theorien.

\subsection{Opportunitätskosten}

In der Neuen Haushaltsökonomik sind Fertilitätsentscheidungen eine Funktion individueller Präferenzen und der Kosten von Kindern, gegeben eine Einkommensrestriktion (Becker 1991; Cigno 1991; Willis 1973). Da Eltern mit zunehmender ,Qualität' und ,Quantität' der Kinder Nutzen erzielen, sind die Kosten von Kindern in dem Modell endogen, d.h. ergeben sich durch das Modell. Diese beinhalten Opportunitätskosten (den Einkommensverlust bei reduziertem Arbeitsangebot), Betreuungskosten (einschließlich der Verfügbarkeit von Kinderbetreuung) sowie Zeitkosten durch das Aufziehen von Kindern (inklusive der häuslichen Arbeitsteilung). Diese Kosten werden zu einem gewissen Anteil durch Einkommensanpassungen der anderen Haushaltsmitglieder ausgeglichen sowie durch Sozialleistungen der Arbeitgeber und Steuern.

Die Neue Haushaltsökonomik betont die Rolle der Löhne der Frauen, welche die Opportunitätskosten von Kindern repräsentieren, als Determinante der Fertilität (z.B. Willis 1973). Frauenlöhne haben sowohl einen (positiven) Einkommenseffekt als auch einen (negativen) Substitutionseffekt auf die Fertilität, und gegenläufige Effekte auf die Ar- 
beitsmarktpartizipation von Frauen. Der Einkommenseffekt besagt, dass mit steigendem Einkommen die Nachfrage nach Kindern steigt mit der Folge eines Anstiegs in der Fertilität. Der Substitutionseffekt impliziert steigende Opportunitätskosten mit steigendem Einkommen, was sich negativ auf die Fertilität auswirkt. Wenn alle Kindererziehungsarbeit von Frauen geleistet wird, wird ein Anstieg in Männerlöhnen einen reinen Einkommenseffekt haben. Der gesamte Effekt eines Anstiegs von Löhnen von Frauen auf die Fertilität ist theoretisch allerdings unklar. Empirisch findet sich fällt bei ansteigendem Frauenlohn (oder andere Maße der Opportunitätskosten von Frauen) die Fertilität (Becker 1991; für eine empirische Übersicht siehe Hotz et al. 1997 und Macunovich 1996). Höhere Frauenlöhne verzögern den Zeitpunkt aller Geburten und reduzieren damit die totale Fertilität (Heckman and Walker 1990). Reduzierte Lohndifferenzen zwischen Männern und Frauen führen zu einer Substitution von Kindererziehungstätigkeiten mit Arbeitsmarktaktivitäten (Galor and Weil 1996). Oder umgekehrt, je höher die Männereinkommen relativ zu den Fraueneinkommen ausfallen, desto eher werden Erwerbstätigkeiten von Frauen zugunsten von Kinderbetreuung aufgegeben.

Arbeitslosigkeit wird von der Neuen Haushaltsökonomik zwar nicht explizit diskutiert; verstanden als null Einkommen impliziert Arbeitslosigkeit theoretisch allerdings einen starken Einkommenseffekt in Haushalten, in denen der Ehemann erwerbstätig ist, während es sowohl einen Einkommens- als auch einen Substitutionseffekt haben sollte, wenn eine erwerbstätige Frau arbeitslos wird (Ahn and Mira 2002). Die empirische Evidenz zeigt allerdings gemischte Resultate: Andersson (2001) berichtet einen stärkeren Einkommenseffekt für individuelle Frauenerwerbstätigkeit in Schweden während der 1980er und 1990er Jahre, während Kravdal (2002) für die Periode 1992-1998 einen leicht stärkeren Substitutionseffekt für individuelle Arbeitslosigkeit auf Erstgeburten in Norwegen, und einen leicht stärkeren Einkommenseffekt für Zweit- und Drittgeburten berichtet.

Butz und Ward (1979) verwenden das Modell der Neuen Haushaltsökonomik, um eine kausale Makrobeziehung zwischen Fertilität und Frauenerwerbstätigkeit zu definieren. In ihrem Modell wird postuliert, dass Fluktuationen in der Fertilität durch einen dominanten Substitutionseffekt von steigenden Frauenlöhnen und damit variierenden Levels von Frauenerwerbsquoten erklärt werden können. Die Partizipationsraten werden als exogen zu den Fertilitätsraten angenommen, wobei sich die Partizipationsraten der Frauen durch den Reservationslohnsatz ergeben. Dieser Reservationslohnsatz ist wiederum determiniert durch das Einkommen des Mannes sowie anderen Charakteristika.

\subsection{Relatives Einkommen}

Easterlins relative Einkommenshypothese ist wie das Modell von Butz und Ward ein Makroansatz zur Erklärung von Fertilität und Frauenerwerbstätigkeit. In dieser Theorie wird das Einkommen des Mannes relativ zu der Elterngeneration in den Mittelpunkt gestellt. Niedrige Fertilität und höhere Frauenerwerbstätigkeit werden in diesem Modell als rationale Entscheidung angesehen, um den relativen ökonomischen Status einer größeren Geburtskohorte zu erhöhen. 
Die Verbindung zwischen höheren Geburtenraten und nachteiligen ökonomischen und sozialen Umständen resultiert in Easterlins Ansatz aus ,Crowding' Mechanismen, die innerhalb von Familie, Schule und Arbeitsmarkt wirken (Easterlin 1980, 1987; vgl. Kapitel $2.2 \mathrm{im}$ vorliegenden Beitrag). In der Theorie von Easterlin wird zunächst das Einkommen des Mannes relativ zu der Elterngeneration als treibende Kraft für Fertilität und Erwerbstätigkeit angesehen. Das Einkommen der Elterngeneration formt ökonomische Aspirationen bei der Nachfolgegeneration. Ein Anstieg im Einkommen relativ zur ökonomischen Aspiration verlagert die Präferenzen von der Erwerbstätigkeit von Frauen hin zu Kindern. Ein Absinken des relativen Einkommens verlagert die Präferenzen von Kindern hin zu steigender Frauenerwerbstätigkeit. ${ }^{2}$

Im kompletten Easterlin-Modell hängt das relative Einkommen von der Größe der jungen Kohorten relativ zu der vorhergehenden Kohorte ab, wobei die Einkommen von Männern und Frauen gleichzeitig gemessen werden. Eine ungewöhnlich große Kohorte junger Erwachsener sieht sich einer größeren Konkurrenz von Gleichaltrigen in Bildungsund Erwerbschancen ausgesetzt, mit nachteiligen Konsequenzen für die Löhne. Gleichzeitig sollten die Löhne der einer kleineren Kohorte angehörenden Elterngeneration relativ hoch sein, wodurch sich hohe ökonomische Aspirationen bei der Nachfolgegeneration bei der Entscheidung ,Erwerbstätigkeit oder Kinder' gebildet haben. Damit steht hinter steigender Erwerbstätigkeit und sinkender Fertilität der Wunsch einer relativ großen Geburtskohorte nach einer Verbesserung des relativen Einkommens, wobei das elterliche Einkommen als Maß der materiellen Aspirationen dient. Empirische Tests von Easterlins Modell werden in den Literaturübersichten von Pampel und Peters (1995) sowie Macunovich (1998) präsentiert. Zusammenfassend unterstützt die Forschung das Konzept des relativen Einkommens; die Ursachen unterschiedlicher materieller Aspirationen scheinen jedoch unklar.

Da weder die Neue Haushaltsökonomik noch die Easterlin-Hypothese die zeitliche Entwicklung und länderspezifische Variation der Entwicklung von Frauenerwerbstätigkeit und Fertilität erklären, ist in den letzten Jahren der ökonomische Ansatz durch die Einbeziehung von Normen und Werten, sozialen Interaktionen sowie institutionellen Rahmenbedingungen erweitert worden (Engelhardt und Prskawetz 2004).

\subsection{Lebensverlaufsperspektive}

Die Lebensverlaufsperspektive ist seit Anfang der 1990er Jahre sowohl bei der Untersuchung demographischer Veränderungen (Diekmann und Weick 1993) als auch bei der Analyse von Arbeitsmarktprozessen (Blossfeld 1990) von vielen Soziologen herangezogen worden. Im Zentrum des Interesses stehen dabei die gesellschaftliche Institutionalisierung und Deinstitutionalisierung zentraler Ereignisse im Lebensverlauf und Auswirkungen auf Verhalten sowie Lebenschancen (für einen Überblick siehe Blossfeld und Huinink 2001). Unseres Erachtens steht hinter der Lebensverlaufsperspektive keine in sich geschlossene Theorie, sondern vielmehr eine Art der Betrachtung des Forschungsgegenstandes. Zur

\footnotetext{
${ }^{2}$ In den ersten theoretischen Betrachtungen waren Frauen jedoch überhaupt nicht vertreten, da Easterlin davon ausging, dass Frauen auf dem Arbeitsmarkt nicht vertreten sind (Easterlin 1978).
} 
Formulierung konkreter Hypothesen werden dann häufig andere Theorien wie die Neue Haushaltsökonomik benutzt.

Im Folgenden werden einige Argumente zu dem Timing und der Inzidenz von Elternschaft aus der Lebensverlaufsperspektive vorgestellt werden. Da eine Elternschaft eine langfristige bindende soziale Beziehung darstellt, müssen eine Reihe biographischer Voraussetzungen erfüllt sein, bevor jemand meint, dafür bereit zu sein. Huinink (2001) nennt in diesem Zusammenhang drei Problemkreise: Perspektivenprobleme, Ressourcenprobleme und Vereinbarkeitsprobleme.

Perspektivenprobleme betreffen die spezifische Lebenssituation junger Menschen. Jugendliche und junge Erwachsene, die in Ausbildung sind und die Grundlagen ihrer beruflichen Laufbahn legen, denken nach der Lebensverlaufsperspektive noch gar nicht ernsthaft über das ,wann' und ,ob' einer Elternschaft nach. Die Ausbildungszeit ist Teil der Lebensphase, in der die Ideen zu den Konturen der zukünftigen Lebensgestaltung erst allmählich entstehen. Solange die Frage nach dem zukünftigen Lebensverlauf nicht beantwortet ist, werden langfristige Bindungen, wie sie eine Eheschließung oder eine Elternschaft typischerweise darstellt, vermieden.

Umgekehrt lässt sich aber auch argumentieren, dass Unsicherheiten hinsichtlich der biographischen Perspektive einen gegenteiligen Effekt haben. Eine Eheschließung oder der Eintritt in eine Elternschaft können als mögliche Strategie zur Lösung von Perspektivenproblemen verstanden werden. Dieser Akt der Selbstbindung hilft bei der Strukturierung des zukünftigen Lebensverlaufs. Teenagerschwangerschaften werden mitunter auf diese Weise begründet (Huinink 2001).

Ressourcenprobleme betreffen die finanzielle Situation junger Menschen, die in der Regel während der Ausbildungszeit finanziell weitgehend von den Eltern abhängig sind. Ein stabiles, eigenständig erwirtschaftetes und hinreichend hohes Einkommen ist aber eine wichtige Grundlage für die Versorgung einer Familie. Während hierfür im traditionellen Familienmodell (,male chauvinist model') allein die Männer verantwortlich waren, vollzieht sich im Zusammenhang mit zunehmender Erwerbsbeteiligung der Frauen ein Wandel. Hinter dem Anstieg der Partizipationsrate wird zum einen ein Mittel zur Befriedigung gestiegener Ansprüche und zum anderen der Wunsch nach Sicherung der wirtschaftlichen Grundlage des eigenen Lebens gesehen. Huinink (2001) sieht sogar eine gesellschaftliche Erwartung oder Norm der eigenen Existenzsicherung von Frauen. Aus diesen Betrachtungen kann man schließen, dass nicht nur die Männer, sondern auch die Frauen zunächst Sicherheit in ihren berufsbezogenen Lebensbereichen erreichen wollen, bevor sie langfristig bindende Entscheidungen in anderen Lebensbereichen, wie in Bezug auf eine Familiengründung, treffen.

Vereinbarkeitsprobleme betreffen die individuelle Zeitökonomie. Der Erwerb eines Ausbildungsabschlusses und die anschließende Erwerbsbeteiligung brauchen mehr oder weniger Lebenszeit, die nicht für die Familienbildung und die Kindererziehung zur Verfügung steht. Eine Elternschaft ist daher erst erstrebenswert, wenn eine Vereinbarkeit zwischen Elternschaft und Erwerbsbeteiligung in Sicht ist. 
Die Inkompatibilität zwischen Erwerbstätigkeit und Familie hat sich in vielen Ländern mit dem Wandel der Industrie- und Berufsstrukturen und dem damit einhergehenden Anstieg von Teilzeitarbeitsmöglichkeiten reduziert (O'Reilly and Fargan 1998). Im internationalen Vergleich zeigen denn auch Länder mit vermehrten Teilzeitarbeitsmöglichkeiten höhere Fertilitätsraten als Länder mit einer geringen Anzahl an Teilzeitarbeitsplätzen (Engelhardt und Prskawetz 2004).

Wie de Laat und Sevilla-Sanz (2003) feststellen, sind Vereinbarkeitsprobleme zwischen Fertilität und Erwerbstätigkeit eine überzeugende Komponente in der Erklärung der positiven Korrelation zwischen Fertilität und Frauenerwerbstätigkeit im internationalen Vergleich. Im Besonderen kann hiermit gut erklärt werden, warum Frauen in einigen Ländern Entscheidungen zugunsten von Fertilität und Erwerbstätigkeit treffen, während in anderen Ländern die Entscheidung Fertilität oder Erwerbstätigkeit lautet. Damit kann auch die Kombination von niedriger Fertilität und hoher Erwerbsbeteiligung sowie hoher Fertilität und hoher Erwerbsbeteiligung begründet werden. Nicht erklärt werden kann hingegen, warum manche Länder bei niedrigen Fertilitätsraten eine geringe Partizipationsrate aufweisen. Autoren wie de Laat und Savilla-Sanz (2003), Bettio und Villa (1998) sowie Di Tomasso (1999) haben argumentiert, dass Unterschiede in Normen bezüglich Familienstrukturen und deren Rolle im wirtschaftlichen Leben zwischen Hoch- und Niedrigfertilitätsländern die spezielle Situation der Länder mit niedriger Fertilität und niedrigen Frauenerwerbsraten zu erklären vermögen (siehe hierzu Engelhardt und Prskawetz 2004).

Perspektiven-, Ressourcen- und Vereinbarkeitsprobleme werden zunehmend später im Lebenslauf gelöst. Daher ist der starke Aufschub des Eintritts in eine Elternschaft in guter Weise begründet. ${ }^{3}$

\section{Ausblick}

Wie wir im vorliegenden Beitrag zeigen konnten, besteht zwischen dem Arbeitsmarkt und der Demographie eine enge Verbindung. So reagiert der Arbeitsmarkt elastisch bezüglich demographischer Veränderungen, und umgekehrt reagieren demographische Prozesse sensibel auf Arbeitsmarktstrukturen sowie Prozesse auf dem Arbeitsmarkt. Dieser Sachverhalt hat denn auch in den letzten Jahren Ökonomen und Demographen angeregt, sich verstärkt mit dieser Thematik zu beschäftigen. Während für Ökonomen die Demographie zumeist auf der rechten Seite der Gleichung auftaucht, verhält es sich bei den Demographen genau umgekehrt. Die enge Verknüpfung beider Gebiete macht es für Wissenschaftler beider Disziplinen jedoch unabdingbar, sich auch jeweils mit dem anderen Metier zu beschäftigen.

Wie unser Überblick gezeigt hat, steckt die Forschung über die Verknüpfung von Arbeitsmarkt und Demographie in vielen Bereichen noch in den Kinderschuhen. Auf vielen Gebieten sind die empirischen Befunde nicht eindeutig (z.B. die Evidenzen zum Modell

\footnotetext{
${ }^{3} \mathrm{Zu}$ einem anderen Ansatz eines biographietheoretischen Ansatzes zum generativen Verhalten siehe Birg et al. (1991).
} 
von Easterlin) bzw. die theoretischen Erklärungen empirischer Beobachtungen unzureichend (z.B. die Erklärung niedriger Fertilität in Niedrigpartizipationsländern). Beide Probleme können prinzipiell durch zwei Faktoren verursacht sein: Die theoretischen Modelle sind nicht hinreichend entwickelt und/oder die empirischen Daten sind nicht ausreichend. In den nächsten Jahren wird sich in beiden Bereichen einiges tun. An den theoretischen Modellen wird an vielen akademischen Instituten derzeit verstärkt gearbeitet, und das internationale SHARE-Projekt (Survey of Health, Ageing and Retirement in Europe) wird neue Daten über das Altern liefern, wodurch wichtige Erkenntnisse über den Zusammenhang von Produktivität und Altern gewonnen werden können. Die Resultate zum Zusammenhang von Demographie und Arbeitsmarkt sind auf jeden Fall nicht nur im akademischen Sinne interessant, sondern insbesondere auch für politische Entscheidungsträger relevant. 


\section{Literatur}

Ahn, Namkee und Pedro Mira. 2002. A Note on the Changing Relationship Between Fertility and Female Employment Rates in Developed Countries. Journal of Population Economics 15: 667-682.

Andersson, Gunnar. 2001. The Impact of Labour-Force Participation on Childbearing Behavior: Pro-Cyclical Fertility in Sweden during the 1980s and the 1990s. European Journal of Population 16: 293-333.

Arnds, Pascal und Holger Bonin. 2003. Gesamtwirtschaftliche Folgen demographischer Alterungsprozesse. S. 131-177 in Arbeit in einer alternden Gesellschaft, Problembereiche und Entwicklungstendenzen der Erwerbssituation Älterer, herausgegeben von Matthias Herfurth, Martin Kohli und Klaus F. Zimmermann. Opladen: Leske+Budrich.

Baltes, Paul B. und Margret M. Baltes. 1989. Erfolgreiches Altern: Eine Perspektive aus psychologischer Sicht. Zeitschrift für Pädagogik 34: 85-105.

Becker, Gary S. 1962. Investment in human capital: A theoretical analysis. Journal of Political Economy 70: 9-49.

Becker, Gary S. 1991. A Treatise on the Family, enl. ed. Cambridge. Mass.: Harvard University Press.

Bender, Stefan, Bert Rürup, Wolfgang Seifert und Werner Sesselmeier. 2000. Migration und Arbeitsmarkt. S. 59-83 in Migrationsreport 2000: Fakten - Analysen - Perspektiven, herausgegeben von Klaus J. Bade und Rainer Münz. Frankfurt am Main: Campus Verlag.

Berger, Mark C. 1989. Demographic Cycles, Cohort Size and Earnings. Demography 26: 311-321.

Bettio, Francesca und Paola Villa. 1998. A Mediterranean Perspective on the Break-Down of the Relationship Between Participation and Fertility. Cambridge Journal of Economics 22: $137-171$.

Birg, Herwig, E.-Juergen Floethmann und Iris Reiter. 1991. Biographische Theorie der demographischen Reproduktion. Frankfurt, NY: Campus.

Blossfeld, Hans-Peter. 1990. Berufsverläufe und Arbeitsmarktprozesse. Ergebnisse sozialstruktureller Längsschnittuntersuchungen. S. 118-145 in Lebensverläufe und sozialer Wandel, herausgegeben von Karl Ulrich Mayer. Opladen: Westdeutscher Verlag.

Blossfeld, Hans-Peter und Johannes Huinink. 2001. Lebensverlaufsforschung als sozialwissenschaftliche Forschungsperspektive. Themen, Konzepte, Methoden und Probleme. BIOS 14(2): 5-31. 
Blien, Uwe und Wolfgang Meyer. 2000. Bevölkerungsdynamik und Arbeitsmarkt. S. 1025-1065 in Handbuch der Demographie II, herausgegeben von Ulrich Mueller, Bernhard Nauck und Andreas Diemann. Berlin: Springer.

Bloom, David E., Richard B. Freeman und Sanders D. Korenman. 1987. The LabourMarket Consequences of Generational Crowding. European Journal of Population 3: 131176.

Börsch-Supan A. 2002. Labor market effects of population aging. MEA Discussion Papers 11-2002, Mannheimer Forschungsinstitut Ökonomie und Demographischer Wandel.

Brewster, Karin L. and Ronald R. Rindfuss. 2000. Fertility and Women's Employment in Industrialized Nations. Annual Review of Sociology 26: 271-296.

Budig, Michelle J. und Paula England. 2001. The Wage Penalty for Motherhood. American Sociological Review 66: 204-225.

Butz, William P. und Michael P. Ward. 1979. The Emergence of Countercyclical U.S. Fertility. American Economic Review 69: 318-328.

Cigno, Alessandro. 1991. Economics of the Family. Oxford: Clarendon Press.

Dahlberg, Susanne und Ruth-Aïda Nahum. 2003. Cohort Effects on Earnings Profiles: Evidence from Sweden. Institute for Future Studies.

de Laat, Joost und Almudena Sevilla-Sanz. 2003. The Unequal Division of Household Labor: Gender Roles and the Puzzle of Positive Correlation Between Fertility and Female Labor-Force Participation. Brown University: unveröffentlichtes Manuskript.

Del Boca, Daniela. 2002. The Effect of Childcare and Part Time Opportunities on Participation and Fertility Decisions in Italy. IZA Discussion Paper No. 427. Bonn: Forschungsinstitut zur Zukunft der Arbeit.

Diekmann, Andreas und Stefan Weick. 1993. Der Familienzyklus als sozialer Prozeß. Berlin: Duncker \& Humblot.

Di Tommaso, Maria L. 1999. A Trivariate Model of Participation, Fertility and Wages: The Italian Case. Cambridge Journal of Economics 23: 623-640.

Easterlin, Richard A. 1980. Birth and Fortune: The Impact of Numbers on Personal Welfare. New York: Basic Books.

Easterlin, Richard A. 1978. What will 1984 be like? Demography 15: 397-421.

Easterlin, Richard A. 1980. Birth and Fortune: The Impact of Numbers on Personal Welfare. New York: Basic Books. 
Easterlin, Richard A. 1987. Birth and Fortune: The Impact of Numbers on Personal Fortune, $2^{\text {nd }}$ Edition. Chicago: University of Chicago Press.

Engelhardt, Henriette, Tomas Kögel und Alexia Prskawetz. 2004. Fertility and Women's Employment: A Macro-Level Time Series Analysis for Developed Countries, 1960-2000. Population Studies 58: 109-120.

Engelhardt, Henriette und Alexia Prskawetz. 2004. On the Changing Correlation Between Fertility and Female Employment Over Space and Time. European Journal of Population 20: $35-62$.

Engelhardt, Henriette und Alexia Prskawetz. 2005. A Pooled Time-Series Analysis on the Relation Between Fertility and Female Employment. Working paper. Wien: Institut für Demographie.

Ermisch, John F. 1988. Fortunes of Birth: The Impact of Generation Size on Relative Earnings of Young Men. Scottish Journal of Political Economy 35: 266-282.

Galor, Oded und David N. Weil. 1996. The Gender Gap, Fertility and Growth. American Economic Review 86: 374-387.

Gauthier, Anne H. 1996. The State and the Family: A Comparative Analysis of Family Policies in Industrialized Countries. Oxford: Clarendon Press.

Gottschalk, Peter. 2001. What Can We Learn from the Cohort Size Literature about the Future Demand for the Greying Baby Boomers? Unpublished paper for a Roundtable on the Demand for Older Workers, Brookings Institution, March 2001.

Gustafsson, Siv. 1981. Male-Female Lifetime Earnings Differentials. S. 235-268 in Studies in Labour Market Behavior: Sweden and the United States, herausgegeben von Gunnar Eliasson, Bertil Holmlund und Frank Stafford. Stockholm, Industrial Institute for Social and Economic Research.

Gustafsson, Siv. 1976. Lönebildning och lönestruktur inom den statliga sektorn (Determination and Structure of Salaries in the Government sector of Sweden), for Industriens Utredningsinstitut (IUI), Uppsala: Almqvist \& Wiksell International.

Heckman, James J. und James R. Walker. 1990. The Relationship between Wages and Income and the Timing and Spacing of Births: Evidence from Swedish Longitudinal Data. Econometrica 58: 1411-1441.

Herfurth, Matthias, Martin Kohli und Klaus F. Zimmermann. 2003. Arbeit in einer alternden Gesellschaft, Problembereiche und Entwicklungstendenzen der Erwerbssituation Älterer. Opladen: Leske+Budrich. 
Hotz, V. Joseph, Jacob A. Klerman und Robert J. Willis. 1997. The Economics of Fertility in Developed Countries. S. 275-347 in Handbook of Population and Family Economics, herausgegeben von Mark R. Rosenzweig und Oded Stark. Amsterdam: Elsevier.

Jann, Ben und Henriette Engelhardt. 2005. Wage Dispersion, Segregation and the Swiss Gender Wage Gap, 1991-2003. Unveröffentlichtes Manuskript, ETH Zürich.

Johnson, Paul und Klaus F. Zimmermann. 1993. Labour Markets in an Ageing Europe. Cambridge: Cambridge University Press

Klevmarken, N. Anders. 1982. On the Stability of Age-Earnings Profiles. Scandinavian Journal of Economics 84: 531-554.

Klevmarken, N. Anders. 1993a. On Ageing and Earnings. S. 151-181 in Labour Markets in an Ageing Europe, herausgegeben von Paul Johnson und Klaus F. Zimmermann. Cambridge University Press.

Klevmarken, N. Anders. 1993b. Demographics and the Dynamics of Earnings. Journal of Population Economics 6: 105-122.

Korenmann Sanders und David Neumark. 1997. Cohort Crowding and Youth Labor Markets: A Cross-National Analysis. NBER Working Paper No. 6031.

Korenman, Sanders und David Neumark. 2000. Cohort Crowding and Youth Labor Market: A cross-national analysis. S. 57-106 in Youth Employment and Joblessness in Advanced Countries, NBER Comparative Labour Market Series, herausgegeben von David G. Blanchflower und Richard B. Freeman. Chicago: University of Chicago Press.

Kravdal, Øystein. 2002. The Impact of Individual and Aggregate Unemployment and Fertility in Norway. Demographic Research, Vol. 6, Article 10. www.demographicresearch.org.

Lillard, Lee A. und Yoram Weiss. 1979. Components of Variation in Panel Earnings Data: American Scientists 1960-70. Econometrica 47: 437-454.

Luckert, Hilmar, Christof Kühnlein und Leonhard Knoll. 1995. Kohorteneffekte auf Niveau und Entwicklung von Arbeitseinkommen im Laufe des Berufslebens - eine empirische Studie. Vierteljahreshefte zur Wirtschaftsforschung 64: 602-623.

Macunovich, Diane A. 1996. A Review of Recent Developments in the Economics of Fertility. S. 91-150 in Household and Family Economics, herausgegeben von Paul L. Menchik. Kluwer: Academic Press.

Macunovich, Diane A. 1998. Fertility and the Easterlin Hypothesis: An Assessment of the Literature. Journal of Population Economics 11: 53-111. 
Macunovich, Diane A. 1999. The Fortunes of One's Birth: Relative Cohort Size and the Youth Labour Market in the U.S. Journal of Population Economics 12: 215-272.

Mincer, Jacob. 1958. Investment in Human Capital and Personal Income Distribution. Journal of Political Economy 66: 281-302.

Mincer, Jacob. 1962. On-the-Job Training: Costs, Returns and Some Implications. Journal of Political Economy 70: 50-79.

Mincer, Jacob. 1970. The Distribution of Labour Income: A Survey with Special Reference to the Human Capital Approach. Journal of Economic Literature 8: 1-26.

Mincer, Jacob. 1974. Schooling, Experience and Earnings. New York: National Bureau of Economic Research.

Mincer, Jacob und Salomon Polacheck. 1974. Family Investments in Human Capital: Earnings of Women. Journal of Political Economy 82: 76-108.

Mincer, Jacob und Haim Ofek. 1982. Interrupted Work Careers. Journal of Human Resources 17: 3-24.

Pampel, Fred C. 2001. The Institutional Context of Population Change. Chicago: The University of Chicago Press.

Pampel, Fred C. und H. Elisabeth Peters. 1995. The Easterlin Effect. Annual Review of Sociology 21: 163-194.

O'Reilly, Jacqueline und Colette Fagan (eds.). 1998. Part-Time Prospects. An International Comparison of Part-time Work in Europe, North America and the Pacific Rim. London, New York: Routledge.

Reskin, Barbara F. und Desise D. Bielby. 2005. A Sociological Perspective on Gender and Career Outcomes. Journal of Economic Perspectives 19: 71-86.

Rindfuss, Ronald R. und Karin L. Brewster. 1996. Childrearing and Fertility. Population and Development Review 22 (Supplement): 258-289.

Rindfuss, Ronald R., Karen Benjamin Guzzo und S. Philip Morgan. 2003. The Changing Institutional Context of Low Fertility. Population Research and Development Review 22: 411-438.

Salthouse, Timothy A. 1991. Theoretical Perspectives on Cognitive Aging. Hillsdale: Erlbaum.

Schmidt, Christoph M. 1993. Ageing and Unemployment. S. 216-254 in Labour Markets in an Ageing Europe, herausgegeben von Paul Johnson und Klaus F. Zimmermann. Cambridge: Cambridge University Press. 
Schultz, Theodore W. 1961. Investment in Human Capital. American Economic Review 51: 1-17.

Shimer, Robert. 2001. The Impact of Young Workers on the Aggregate Labor Market. Quarterly Journal of Economics 116(3): 969-1007.

Skirbekk, Vegard. 2004. Age and Individual Productivity: A Literature Survey. Vienna Yearbook of Population Research, im Erscheinen.

Stapeltone, David C. und Douglas J. Young. 1988. Educational Attainment and Cohort Size. Journal of Labor Economics 6: 330-361.

Welch, Finis. 1979. Effects of Cohort Size on Earnings: The Baby Boom Babies' Financial Bust. Journal of Political Economics 87: 65-97.

Wright, Robert E. 1989. The Easterlin Hypothesis and European Fertility Rates. Population Development Review 15: 107-122.

Wright, Robert E. 1991. Cohort Size and Earnings in Great Britain. Journal of Population Economics 4: 295-305.

Willis, Robert J. 1973. A New Approach to the Economic Theory of Fertility Behavior. Journal of Political Economy 81: S14-S64.

Zimmermann, Klaus F. 1991. Ageing in the Labor Market: Age Structure, Cohort Size and Unemployment. Journal of Population Economics 4: 177-200. 


\section{VIENNA INSTITUTE OF DEMOGRAPHY}

\section{Working Papers}

Winkler-Dworak, Maria. 2003. Food Security, Fertility Differentials and Land Degradation in Sub-Saharan Africa: A Dynamic Framework. VID Working Papers 01/2003. Vienna Institute of Demography.

Frejka, Tomas and Jean-Paul Sardon. 2003. Fertility Austria: Past, Present and the Near Future. VID Working Papers 02/2003. Vienna Institute of Demography.

Sanderson, Warren C., Sergei Scherbov, Brian C. O'Neill, and Wolfgang Lutz. 2003. Conditional probabilistic population forecasting. VID Working Papers 03/2003. Vienna: Vienna Institute of Demography.

Fliegenschnee, Katrin, Anne Goujon, and Wolfgang Lutz. 2004. Neue demographische Szenarien zur Zukunft der Evangelischen Kirche in Österreich. VID Working Papers 01/2004. Vienna: Vienna Institute of Demography.

Engelhardt, Henriette. 2004. Fertility Intentions and Preferences: Effects of Structural and Financial Incentives and Constraints in Austria. VID Working Papers 02/2004. Vienna: Vienna Institute of Demography.

Prskawetz, Alexia, Tomas Kögel, Warren C. Sanderson, and Sergei Scherbov. 2004. The Effects of Age Structure on Economic Growth: An Application of Probabilistic Forecasting to India. VID Working Papers 03/2004. Vienna: Vienna Institute of Demography.

Schwarz, Franz. 2004. Analysis of Inequalities in Waiting Time at the Visit to the Physician using Regression Modeling for Duration Data. VID Working Papers 04/2004. Vienna: Vienna Institute of Demography.

Sobotka, Tomáš, Maria Winkler-Dworak, Maria Rita Testa, Wolfgang Lutz, Dimiter Philipov, Henriette Engelhardt, and Richard Gisser. 2005. Monthly Estimates of the Quantum of Fertility: Towards a Fertility Monitoring System in Austria. VID Working Papers 01/2005. Vienna: Vienna Institute of Demography.

Engelhardt, Henriette und Alexia Prskawetz. 2005. Arbeitsmarkt und Demographie VID Working Papers 02/2005. Vienna: Vienna Institute of Demography.

Heiland, Frank, Alexia Prskawetz, and Warren C. Sanderson. 2005. Do the More-Educated Prefer Smaller Families? VID Working Papers 03/2005. Vienna: Vienna Institute of Demography.

Kim, Jungho. 2005. Learning by Doing and Learning from Others in Contraceptive Technology. VID Working Papers 04/2005. Vienna: Vienna Institute of Demography.

The Vienna Institute of Demography Working Paper Series receives only limited review. Views or opinions expressed herein are entirely those of the authors. 